Old Dominion University

ODU Digital Commons

Electrical \& Computer Engineering Faculty

Publications

Electrical \& Computer Engineering

2020

\title{
Efficacy of Radiomics and Genomics in Predicting TP53 \\ Mutations in Diffuse Lower Grade Glioma
}

Zeina A. Shboul

Old Dominion University, zshbo001@odu.edu

Khan Iftekharuddin

Old Dominion University, kiftekha@odu.edu

Follow this and additional works at: https://digitalcommons.odu.edu/ece_fac_pubs

Part of the Electrical and Computer Engineering Commons, Nervous System Commons, and the Neurology Commons

\section{Original Publication Citation}

Shboul, Z., \& Iftekharuddin, K. (2020). Efficacy of radiomics and genomics in predicting TP53 mutations in diffuse lower grade glioma. In Krol, A. and Gimi, B.S. (Eds.), Medical Imaging 2020: Biomedical Applications in Molecular, Structural and Functional Imaging, Houston, Texas, U.S.A. 113170Y (1-7). SPIE. https://doi.org/10.1117/12.2550764

This Conference Paper is brought to you for free and open access by the Electrical \& Computer Engineering at ODU Digital Commons. It has been accepted for inclusion in Electrical \& Computer Engineering Faculty Publications by an authorized administrator of ODU Digital Commons. For more information, please contact digitalcommons@odu.edu. 


\section{Efficacy of radiomics and genomics in predicting TP53 mutations in diffuse lower grade glioma}

Shboul, Zeina, Iftekharuddin, Khan

Zeina A. Shboul, Khan M. Iftekharuddin, "Efficacy of radiomics and genomics in predicting TP53 mutations in diffuse lower grade glioma," Proc. SPIE 11317, Medical Imaging 2020: Biomedical Applications in Molecular, Structural, and Functional Imaging, 113170Y (28 February 2020); doi: $10.1117 / 12.2550764$

SPIE. Event: SPIE Medical Imaging, 2020, Houston, Texas, United States 


\title{
Efficacy of Radiomics and Genomics in Predicting TP53 Mutations in Diffuse Lower Grade Glioma
}

\author{
Zeina A. Shboul and Khan M. Iftekharuddin \\ The authors are with the Vision Lab in the Department of Electrical and Computer \\ Engineering, Old Dominion University, Norfolk, VA 23529
}

\begin{abstract}
An updated classification of diffuse lower-grade gliomas is established in the 2016 World Health Organization Classification of Tumors of the Central Nervous System based on their molecular mutations such as TP53 mutation. This study investigates machine learning methods for TP53 mutation status prediction and classification using radiomics and genomics features, respectively. Radiomics features represent patients' age and imaging features that are extracted from conventional MRI. Genomics feature is represented by patients' gene expression using RNA sequencing. This study uses a total of 105 LGG patients, where the patient dataset is divided into a training set (80 patients) and testing set (25 patients). Three TP53 mutation prediction models are constructed based on the source of the training features; TP53-radiomics model, TP53-genomics model, and TP53-radiogenomics model, respectively. Radiomics feature selection is performed using recursive feature selection method. For genomics data, EdgeR method is utilized to select the differentially expressed genes between the mutated TP53 versus the non-mutated TP53 cases in the training set. The training classification model is constructed using Random Forest and cross-validated using repeated 10 -fold cross validation. Finally, the predictive performance of the three models is assessed using the testing set. The three models, TP53-Radiomics, TP53-RadioGenomics, and TP53-Genomics, achieve a predictive accuracy of $0.84 \pm 0.04,0.92 \pm 0.04$, and $0.89 \pm 0.07$, respectively. These results show promise of non-invasive MRI radiomics features and fusion of radiomics with genomics features for prediction of TP53.
\end{abstract}

Keywords: Glioma grading, TP53 mutation prediction and classification, Radiomics, Genomics, Random Forest.

\section{INTRODUCTION}

Diffuse or infiltrative lower-grade gliomas (LGG) are a Central Nervous System (CNS) brain tumor that include the World Health Organization (WHO) Grade II and III gliomas [1, 2]. Infiltrative LGG arise from the glial cells in the nervous tissues of the CNS [3, 4]. The histology classes of diffuse LGG include astrocytoma, oligodendroglioma, and oligoastrocytoma. However, an updated classification of diffuse LGG is established based on their genetic mutations (such as isocitrate dehydrogenase (IDH) mutation and tumor protein 53 (TP53) mutation) and their histological type [1]. TP53 mutation is a molecular mutation that usually occurs in a younger patient with astrocytoma $[5,6]$. TP53 is a well-known tumor suppressor gene that encodes p53 protein and involves in tumor survival, proliferation, and invasion [7]. A study by Ohgaki et. al, [8] with 715 diagnosed cases of glioblastoma (WHO Grade IV glioma) reveals that mutated TP53 is predictive of longer survival.

Identifying the presence of certain mutations such as TP53 requires molecular profiling using invasive methods by obtaining tumor tissue sample with an increase in the proliferation and neovascularization [9]. Then, Immunohistochemistry (IHC) staining is used to detect missense mutations. Recently, several studies in the literature have proposed the use of RNA sequencing to determine TP53 functional status [10]. However, such invasive methods may also be accompanied by high cost, morbidity, and different difficulties [11]. Therefore, in this work, we investigate different machine learning methods for classification and prediction of TP53 mutation in diffuse LGG based on radiomics, genomics, and fused radiogenomics and compare the performance of these prediction models.

Radiomics are quantitative features that are extracted from volumes of interest in radiology imaging. These quantitative features offer valuable information regarding intensity, shape, size, volume, and texture, and can be associated with glioma clinical outcomes and survival prediction [12-17]. For example Gutman et al. [18], associate three volumetric imaging features with a genetic mutation, survival prediction and Verhaak subtypes [19]. In another study by Shboul et al. [17], the authors investigate the efficacy of using non-invasive quantitative texture features extracted from structural MRI to predict the progression of lower-grade glioma. Compared with the radiomic features, RNA counts data (i.e. genomics) describe the gene expression by counting the relative frequency of the RNA reads

Medical Imaging 2020: Biomedical Applications in Molecular, Structural, and Functional Imaging, edited by Andrzej Krol, Barjor S. Gimi, Proc. of SPIE Vol. 11317, 113170Y · @ 2020 SPIE · CCC code: 1605-7422/20/\$21 · doi: 10.1117/12.2550764 
mapping to one gene [20]. Because RNA is count data, in this study, we log-transformed the differentially expressed genes to normality to use them in machine learning models [21,22].

The proposed work utilizes radiomics and genomics in machine learning modeling to predict TP53 mutations. The proposed work ascertain the efficacy of non-invasive MRI rasdiomics to predict TP53 mutation status when compared to genomics data. The dominant approach in identifying TP53 mutations besides RNA sequencing in literature requires viable tumor tissue that involves invasive tissue sampling. Our results show the efficacy of using either radiomics or radiomiocs fused with the genomics in predicting TP53 mutation status when compared to genomics model alone.

\section{METHOD}

In this study, we investigate TP53 mutation prediction based on a) radiomics (patient age at the diagnosis and imaging features extracted from Magnetic Resonance Imaging (MRI)), b) genomics (gene expression using RNA sequencing), c) and fused radiogenomics (illustrated in Figure 1). First, optimum radiomics are selected using recursive feature selection (RFS), and the top five differentially expressed genes are selected using EdgeR method [23]. Then, the optimum radiomics and the top differentially expressed genes are utilized along using a Random Forest (RF) classifier to construct and train the three different TP53 mutations models: TP53-Radiomics, TP53-Genomics, and TP43RadioGenomics model, respectively. The TP53 mutation models are evaluated using repeated 10-fold CrossValidation (CV). Finally, we assess the predictive performances of these models in predicting TP53 mutation status using the testing dataset.

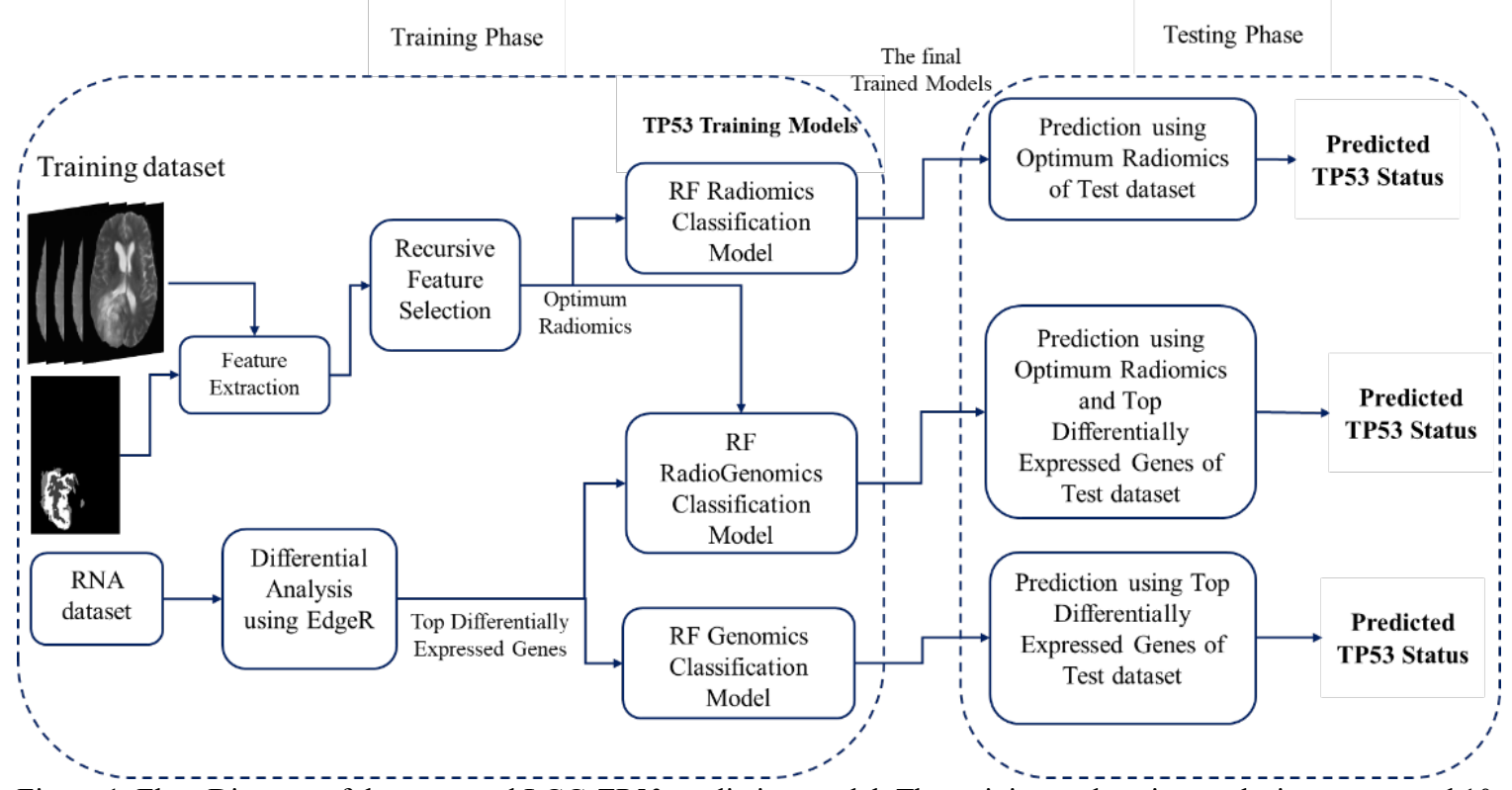

Figure 1. Flow Diagram of the proposed LGG TP53 prediction model. The training and testing analysis are repeated 10 times independently.

\subsection{Dataset}

A total of 105 pre-operative LGG patients described in [24-26] is used to evaluate and asses the proposed models in Figure 1. Four modalities of the MRI are provided with the dataset (T1, T1Gd, T2, FLAIR) along with their segmented tumor. Clinical data and RNA counts data are downloaded from the Genomic Data Commons Data Portal (https://portal.gdc.cancer.gov/). Clinical data are de-identified following the HIPAA standard. The range of the patients' age at the diagnosis is $20-75$ years and the median and the mean age are 46 years. Fifty-five cases harbor TP53 mutations while fifty patients are without TP53 mutations (i.e. wild-type).

\subsection{Extraction of radiomics}

Radiomics are quantitative imaging texture features that are extracted from raw MRI (T1, T1Gd, T2, and FLAIR) sequences of the tumor, five Texton features [27], and multifractal features [28, 29], and the age of the patient at the 
diagnosis. Radiomics features include the histogram, the co-occurrence matrix, the neighborhood gray-tone difference matrix, and the Size Zone Matrix [17, 30].

Also, we utilize volumetric features that are computed as follows: the volume of the whole tumor, the volume of the whole tumor divided by the volume of the brain, the volume of sub-regions (edema, enhance tumor, and necrosis) divided by the volume of the whole tumor, and divided by the volume of the brain, the volumes of the enhance tumor and necrosis divided by the volume of the edema, the combined volume of the edema and enhance tumor, the volume of the edema divided by the combined volume of enhancing tumor and necrosis, and the volume of the necrosis from the combined volume of the edema and enhance tumor.

Additionally, another nine area property features are extracted from the tumor from $x, y$, and $z$-axes. The properties are area, centroid, perimeter, major axis length, minor axis length, eccentricity, orientation, solidity, and extent.

\subsection{Machine learning models for TP53 prediction in LGG}

Three TP53 mutation prediction models are constructed based on the source of the training features; TP53-radiomics model, TP53-genomics model, and TP53-radiogenomics model, respectively. Figure 1 illustrates the complete flow diagram of the three TP53 mutation ML prediction models.

In this study, the dataset is divided into training and testing datasets. Eighty patients are used in the training dataset and the remaining (twenty-five patients) are used in the testing dataset. Feature selection and Random Forest [31] are utilized to construct and train the three different TP53 mutation prediction models. Repeated 10-fold cross-validation is utilized to cross-validate and tune the RF parameters. Radiomics feature selection is performed using recursive feature selection (RFS). RFS is constructed based on RF and is performed by maximizing the ROC metric. In RFS, radiomics are trained using RF, ranked bases on their importance, and then the least important radiomics are removed. The step of training RF using the remaining radiomics and ranking them is repeated recursively until the radiomics' set that maximizes the ROC is reached.

Genomics represented by RNA counts data is the other source of features in this work. EdgeR [23] is exploited to select the top differentially expressed genes (i.e. most important). In EdgeR, the negative binomial distribution is used to model RNA counts. EdgeR assumes that the variance of RNA counts depends on two dispersion parameters; common and individual. Once the negative binomial is fitted and dispersion parameters are obtained, differential expression analysis is performed using either the likelihood ratio test or F-test to find the top differentially expressed genes. Then the top differentially expressed genes are log-transformed into normality and fed into RF to build TP53Genomics and TP53-RadioGenomics.

Moreover, the prediction performance is tested using the testing datasets. The prediction performance is assessed using the accuracy (Acc), balanced accuracy (B. Acc), sensitivity (Sens.), specificity (Spec.), negative predictive value (NPV), and positive predictive value (PPV).

Finally, a comparison of the performance between the three TP53 mutation models is conducted using Analysis of Variance (ANOVA) method. If the $p-$ value of the ANOVA test is less than 0.05 , a statistically significant difference is accepted. Association between TP53 mutation status and the optimum radiomics and the top differentially expressed genes is assessed using ANOVA.

\section{RESULTS}

The RFS method offers the most frequent optimum radiomics features as follows: the age, information correlation, and the low-gray level zone emphasis. EdgeR offers the top differentially expressed genes, and the gene that is the most occurred (in the ten training splits) is TEC gene. Figure 2 illustrates the distribution of the most frequent optimum features and the distribution of the TEC gene expression. Younger ages, low values of low-gray level zone emphasis, and high values of information correlation are significantly associated with mutated TP53 with $p-$ value $<0.05$. Low values of TEC RNA expression are significantly correlated with mutated TP53 with $p-$ value $<0.05$.

Moreover, our analysis shows that the patients' age (at the diagnosis) carries a Hazard Ratio [HR] of 1.07 (95\% CI, 1.03-1.11, likelihood ratio test $p$-value $<0.05$ ). Besides, patients age greater than the median age of 46 years carries a HR of 3.15 (95\% CI, 1.39-7.16, likelihood ratio test $p$-value $<0.05)$ and a median survival of 62 months vs. 94.5 months for patients with age less than 46 years as illustrated in Figure 3a. Unlike patients age at the diagnosis, the optimum radiomics of information correlation and the low gray level zone emphasis and the TEC gene do not show an association with overall survival. Additionally, our analysis reveals that TP53 mutation status does not associate 
with the overall survival (likelihood ratio test $p$-value $=0.1$ ) as illustrated in Figure $3 \mathrm{~b}$. However, as shown in Table 1, a chi-square test reveals that mutated TP53 and patients with ages less than 46 are significantly associated with $p$ value $<0.05$, whereas WT TP53 status is significantly associated with patients with ages greater than 46 .

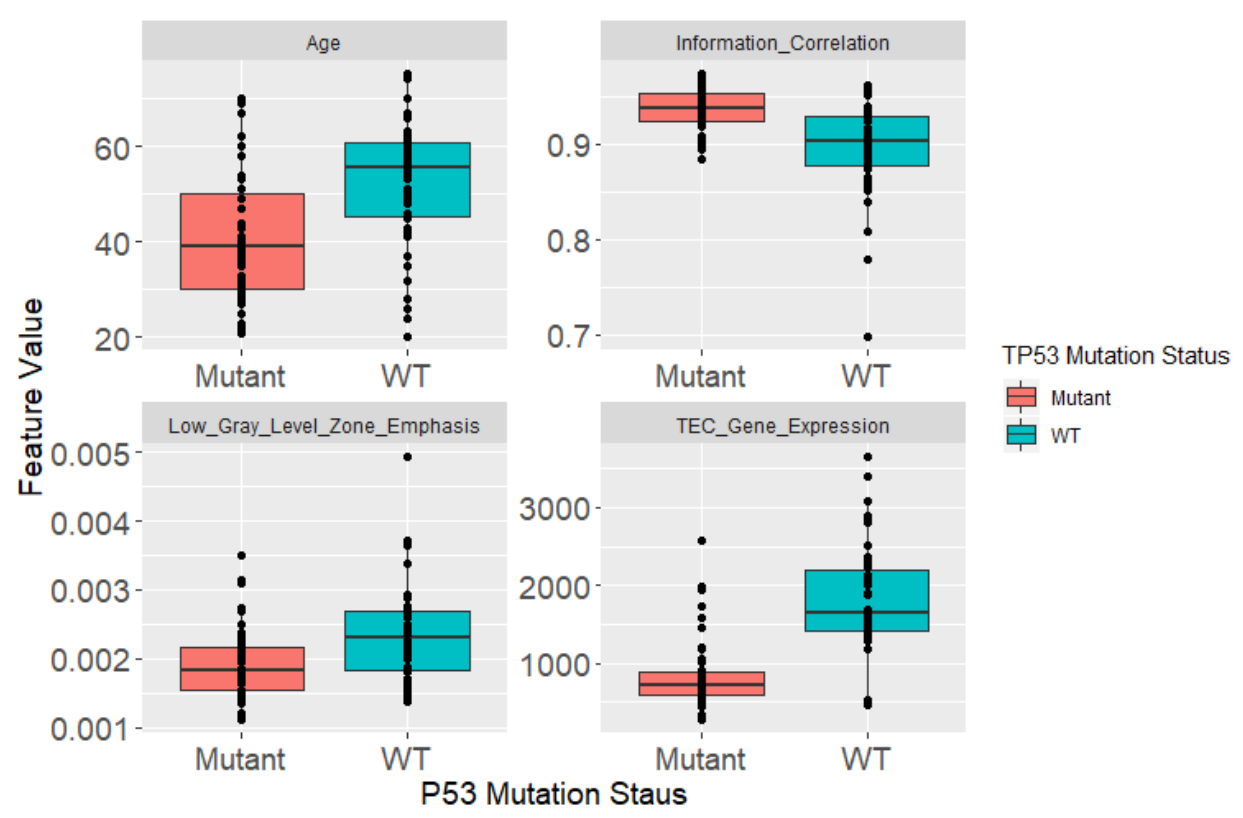

Figure 2. Feature distribution of the optimum radiomics and the top differentially expressed gene in the 10 training sets in discriminating mutated TP53 (Mutant) and not mutated TP53 (i.e. Wild Type (WT)).

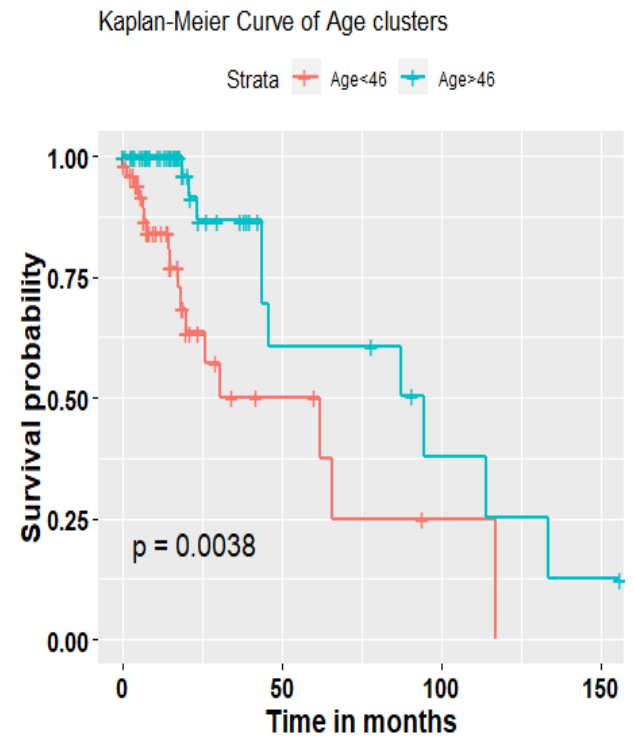

(a)

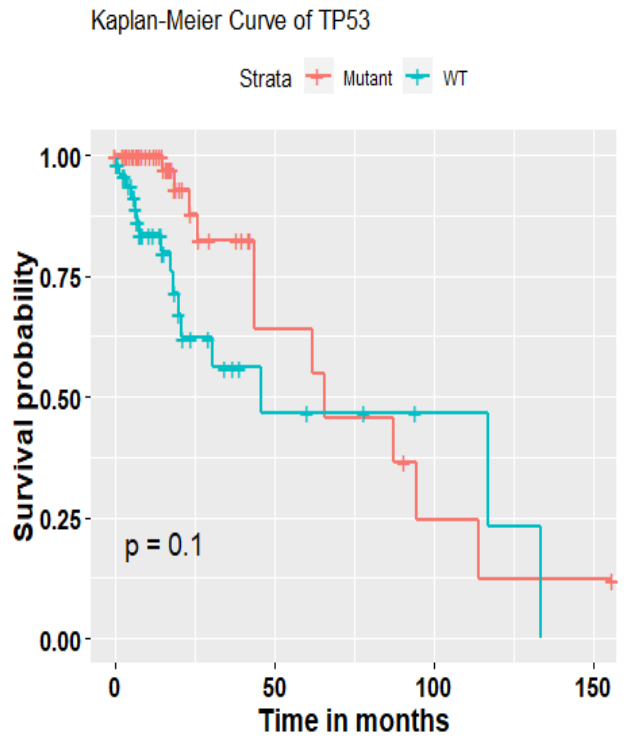

(b)

Figure 3. Kaplan Meier curve of a) patients' age clustered into two groups (age less than 46 years and age greater than 46 years), and b) TP53 mutation status. 
Table 1. TP53 mutation distribution based on the patients' age in the dataset.

\begin{tabular}{|c|c|c|}
\hline & \multicolumn{2}{|c|}{ TP53 mutation Status } \\
\hline Age groups & Mutant & WT \\
\hline Patients' age less than 46 & 39 & 14 \\
\hline Patients' age greater than 46 & 16 & 36 \\
\hline
\end{tabular}

Figure 4 demonstrates the performance of the three TP53 mutation prediction models using the 10 testing sets. These values represent the mean \pm standard deviation of the performance of the 10 repetitions using the testing sets. As illustrated in Figure 4, TP53-Radiomics model achieves an accuracy of $0.84 \pm 0.04$, a sensitivity of $0.87 \pm 0.08$, a specificity of $0.82 \pm 0.09$, and balanced accuracy of $0.84 \pm 0.04$. The performance of TP53-RadioGenomics model (with an accuracy of $0.92 \pm 0.04$, a sensitivity of $0.92 \pm 0.05$, a specificity of $0.93 \pm 0.08$, and balanced accuracy of $0.92 \pm 0.05$ ) outperforms the performance of the TP53-Radiomics models significantly (ANOVA test, $p$-value $<0.05$ ) as shown in Table 2 and Figure 4. Note the performance of the TP53-RadioGenomics model is better than the performance of the TP53-Genomics models (with an accuracy of $0.89 \pm 0.07$, a sensitivity of $0.89 \pm 0.07$, a specificity of $0.89 \pm 0.13$, and balanced accuracy of $0.89 \pm 0.07$ ), however, the improvements of the TP53-RadioGenomics is not significant (ANOVA test, $\mathrm{p}-$ value $>0.05$ ) as shown in Table 2 and Figure 4.

Table 2. The ANOVA Probability value ( $p$-value) of performance of the difference between TP53 mutation prediction models. Note, $p$-value $<0.05$ indicates statistically significant difference.

\begin{tabular}{|c|c|c|c|c|c|c|}
\hline TP53 models comparison & Accuracy & $\begin{array}{c}\text { Balanced } \\
\text { Accuracy }\end{array}$ & $\begin{array}{c}\text { Negative } \\
\text { Predictive } \\
\text { Value }\end{array}$ & $\begin{array}{c}\text { Positive } \\
\text { Predictive } \\
\text { Value }\end{array}$ & Sensitivity & Specificity \\
\hline Radiomics VS. RadioGenomics & 0.000 & 0.000 & 0.051 & 0.004 & 0.094 & 0.010 \\
\hline Radiomics VS. Genomics & 0.085 & 0.083 & 0.418 & 0.083 & 0.517 & 0.147 \\
\hline RadioGenomic VS. Genomics & 0.251 & 0.260 & 0.290 & 0.503 & 0.295 & 0.504 \\
\hline
\end{tabular}

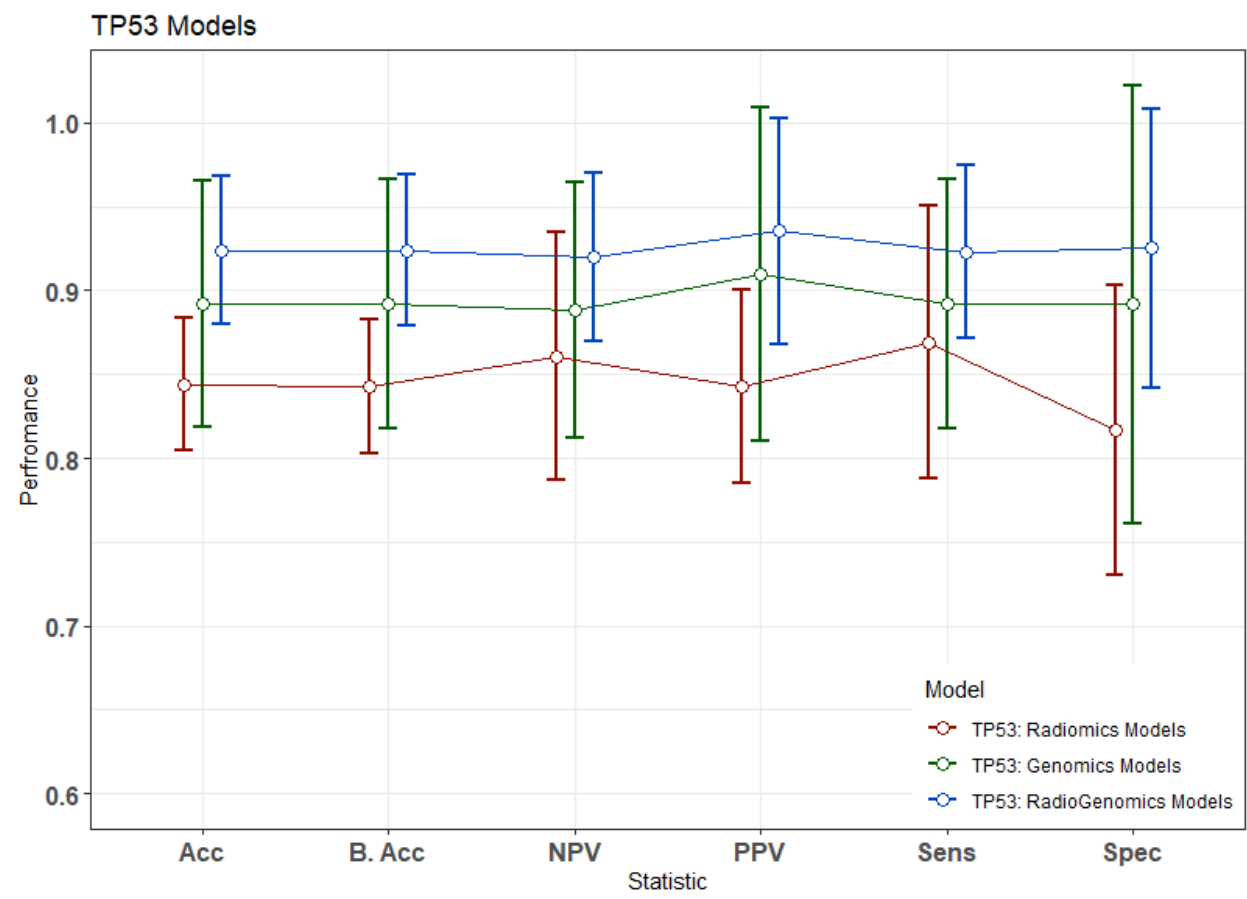

Figure 4. The performance of the TP53 mutation prediction models using 10 different testing splits. Error bar represents two standard deviations. "Acc" refers to accuracy, "B. Acc" refers to balanced accuracy, "NPV" refers to the negative predictive value, "PPV" refers to the positive predictive value, "Sens" refers to sensitivity, and "Spec" refers to specificity. 


\section{CONCLUSION}

This study proposes multiple ML models for TP53 mutation status prediction based on non-invasive radiomics when compared to invasive genomics. Radiomics represent the patients' age and extracted imaging features from the conventional raw and different characterization of MRI modalities (T1, T1Gd, T2, and Flair). Genomics is represented by RNA counts data in this study. Radiomics features selection is performed using RFS whereas the top differentially expressed genes are selected using EdgeR. A total of 105 LGG patients are randomly divided into 10 paired training (80 patients) and testing sets (25 patients). Based on the source of the training features, we construct three TP53 mutation prediction models; the TP53-radiomics model, the TP53-genomics model, and TP53-radiogenomics model, respectively. The training classification of the three models is developed using the Random Forest. Ten-fold crossvalidation is utilized to cross-validate and tune the RF parameters. Finally, the predictive performance of the three models is assessed using the testing set.

Our analysis reveals that age, information correlation, and the low-gray level zone emphasis show significance in predicting TP53 mutation status in the TP53-radiomics model. TEC gene is the most important gene that is used to predict TP53 mutation status in the TP53-Genomics model. Moreover, our TP53-Radiomics model achieves an accuracy of $0.84 \pm 0.04$, a sensitivity of $0.87 \pm 0.08$, a specificity of $0.82 \pm 0.09$, and balanced accuracy of $0.84 \pm 0.04$. In comparison, in a recent study by $\mathrm{Li}$ et. al, [32], the authors propose a TP53 mutation prediction model based on radiomics features. The authors use 180 LGG patients in the training set and 92 patients in the test set. Their proposed method utilize LASSO as a feature selection and SVM. Their proposed method has achieved an AUC of $76.3 \%$, a sensitivity $62.2 \%$, a specificity $85.1 \%$, and an accuracy of $70.7 \%$, in the validation set data. When comparing the performance of our TP53-Radiomics model and the performance of Li et. al, [32], our TP53-Radiomics model achieves better accuracy and sensitivity.

As expected, the TP53-Gadiomics model achieves better performance than the TP53-Radiomics model. However, the TP53-Radiomics model offers comparable performance and the TP53-RadioGenomics model outperforms the TP53Radiomics model significantly. These results suggest efficacy of using conventional MRI as predictive biomarker for TP53 mutation prediction.

\section{ACKNOWLEDGMENTS}

This work was partially funded by NIBIB/NIH grant\# R01 EB020683.

\section{REFERENCES}

[1] Louis, D. N., Perry, A., Reifenberger, G. et al., "The 2016 World Health Organization classification of tumors of the central nervous system: a summary," Acta neuropathologica, 131(6), 803-820 (2016).

[2] Louis, D. N., Ohgaki, H., Wiestler, O. D. et al., "The 2007 WHO classification of tumours of the central nervous system," Acta neuropathologica, 114(2), 97-109 (2007).

[3] Brat, D. J., Verhaak, R. G., Aldape, K. D. et al., "Comprehensive, integrative genomic analysis of diffuse lower-grade gliomas," New England Journal of Medicine, 372(26), 2481-2498 (2015).

[4] Zong, H., Verhaak, R. G., and Canoll, P., "The cellular origin for malignant glioma and prospects for clinical advancements," Expert review of molecular diagnostics, 12(4), 383-394 (2012).

[5] Sarkar, C., Karak, A. K., Nath, N. et al., "Apoptosis and proliferation: correlation with p53 in astrocytic tumours," Journal of neuro-oncology, 73(2), 93-100 (2005).

[6] Watanabe, K., Tachibana, O., Sato, K. et al., "Overexpression of the EGF receptor and p53 mutations are mutually exclusive in the evolution of primary and secondary glioblastomas," Brain pathology, 6(3), 217223 (1996).

[7] Muller, P. A. J., and Vousden, K. H., "p53 mutations in cancer," Nature Cell Biology, 15, 2 (2013).

[8] Ohgaki, H., Dessen, P., Jourde, B. et al., "Genetic pathways to glioblastoma: a population-based study," Cancer research, 64(19), 6892-6899 (2004).

[9] Jalbert, L. E., Elkhaled, A., Phillips, J. J. et al., "Metabolic profiling of IDH mutation and malignant progression in infiltrating glioma," Scientific reports, 7, 44792 (2017).

[10] Troester, M. A., Herschkowitz, J. I., Oh, D. S. et al., "Gene expression patterns associated with p53 status in breast cancer," BMC cancer, 6(1), 276 (2006). 
[11] Hakkarainen, T. W., Kopari, N. M., Pham, T. N. et al., "Necrotizing soft tissue infections: review and current concepts in treatment, systems of care, and outcomes," Current problems in surgery, 51(8), 344 (2014).

[12] Tsien, C., Galbán, C. J., Chenevert, T. L. et al., "Parametric response map as an imaging biomarker to distinguish progression from pseudoprogression in high-grade glioma," Journal of Clinical Oncology, 28(13), 2293 (2010).

[13] Hamstra, D. A., Galbán, C. J., Meyer, C. R. et al., "Functional diffusion map as an early imaging biomarker for high-grade glioma: correlation with conventional radiologic response and overall survival," Journal of clinical oncology, 26(20), 3387 (2008).

[14] Hamstra, D. A., Chenevert, T. L., Moffat, B. A. et al., "Evaluation of the functional diffusion map as an early biomarker of time-to-progression and overall survival in high-grade glioma," Proceedings of the National Academy of Sciences, 102(46), 16759-16764 (2005).

[15] Moffat, B. A., Chenevert, T. L., Lawrence, T. S. et al., "Functional diffusion map: a noninvasive MRI biomarker for early stratification of clinical brain tumor response," Proceedings of the national academy of sciences, 102(15), 5524-5529 (2005).

[16] Shboul, Z. A., Vidyaratne, L., Alam, M. et al., "Glioblastoma and Survival Prediction." International MICCAI Brainlesion Workshop 358-368.

[17] Shboul, Z. A., and Iftekharuddin, K. M., "Prediction of low-grade glioma progression using MR imaging." Proc. SPIE 10950, 1095049.

[18] Gutman, D. A., Cooper, L. A., Hwang, S. N. et al., "MR imaging predictors of molecular profile and survival: multi-institutional study of the TCGA glioblastoma data set," Radiology, 267(2), 560-569 (2013).

[19] Verhaak, R. G., Hoadley, K. A., Purdom, E. et al., "Integrated genomic analysis identifies clinically relevant subtypes of glioblastoma characterized by abnormalities in PDGFRA, IDH1, EGFR, and NF1," Cancer cell, 17(1), 98-110 (2010).

[20] Kukurba, K. R., and Montgomery, S. B., "RNA sequencing and analysis," Cold Spring Harbor Protocols, 2015(11), pdb. top084970 (2015).

[21] McDonald, J. H., [Handbook of biological statistics] sparky house publishing Baltimore, MD, (2009).

[22] Law, C. W., Chen, Y., Shi, W. et al., "voom: Precision weights unlock linear model analysis tools for RNA-seq read counts," Genome biology, 15(2), R29 (2014).

[23] Robinson, M. D., McCarthy, D. J., and Smyth, G. K., "edgeR: a Bioconductor package for differential expression analysis of digital gene expression data," Bioinformatics, 26(1), 139-140 (2010).

[24] Menze, B. H., Jakab, A., Bauer, S. et al., "The multimodal brain tumor image segmentation benchmark (BRATS)," IEEE transactions on medical imaging, 34(10), 1993-2024 (2015).

[25] Bakas, S., Akbari, H., Sotiras, A. et al., "Advancing the cancer genome atlas glioma MRI collections with expert segmentation labels and radiomic features," Scientific data, 4, 170117 (2017).

[26] Bakas, S., Akbari, H., Sotiras, A. et al., "Segmentation Labels and Radiomic Features for the Pre-operative Scans of the TCGA-LGG collection," The Cancer Imaging Archive, (2017).

[27] Leung, T., and Malik, J., "Representing and recognizing the visual appearance of materials using threedimensional textons," International journal of computer vision, 43(1), 29-44 (2001).

[28] Islam, A., Iftekharuddin, K. M., Ogg, R. J. et al., "Multifractal modeling, segmentation, prediction, and statistical validation of posterior fossa tumors." Proc. SPIE 6915, 69153C.

[29] Reza, S., and Iftekharuddin, K., "Multi-fractal texture features for brain tumor and edema segmentation." Proc. SPIE 9035, 903503.

[30] Vallières, M., Freeman, C. R., Skamene, S. R. et al., "A radiomics model from joint FDG-PET and MRI texture features for the prediction of lung metastases in soft-tissue sarcomas of the extremities," Physics in medicine and biology, 60(14), 5471 (2015).

[31] Breiman, L., [Random forests], (2001).

[32] Li, Y., Qian, Z., Xu, K. et al., "MRI features predict p53 status in lower-grade gliomas via a machinelearning approach," NeuroImage: Clinical, 17, 306-311 (2018). 